\title{
Correlating ZnSe Quantum Dot Absorption with Particle Size and
}

\section{Concentration}

Reyhaneh Toufanian ${ }^{1}$, Xingjian Zhong ${ }^{2}$, Joshua C. Kays ${ }^{2}$, Alexander M. Saeboe ${ }^{1}$, Allison

M. Dennis ${ }^{1,2, *}$

${ }^{1}$ Division of Materials Science and Engineering, Boston University, Boston, MA 02215 USA

${ }^{2}$ Department of Biomedical Engineering, Boston University, Boston, MA 02215 USA

*aldennis@bu.edu
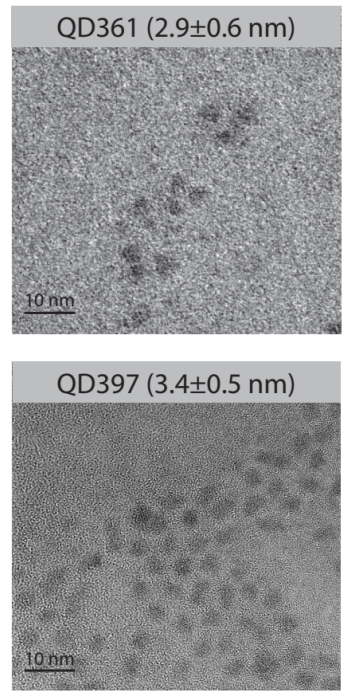
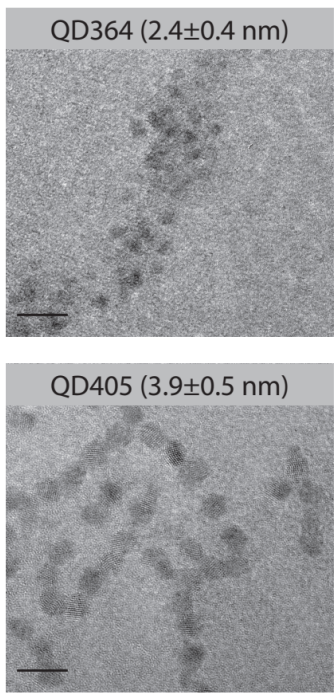
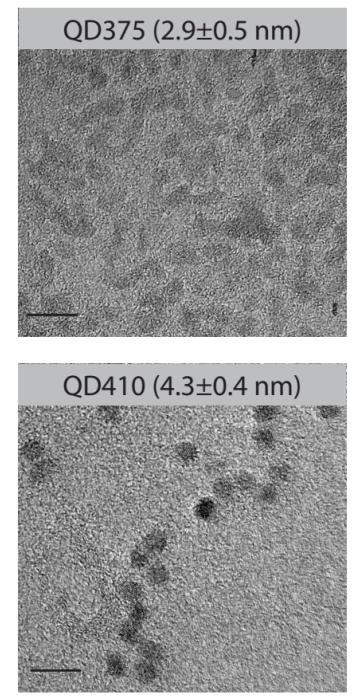
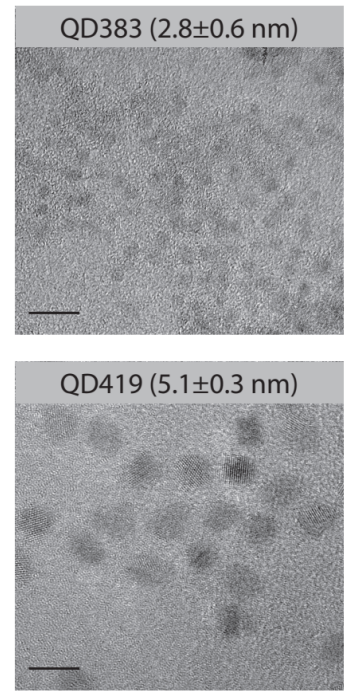

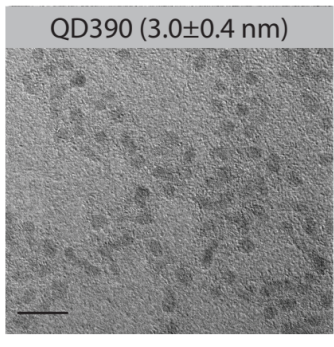

QD422 (5.5 $\pm 0.6 \mathrm{~nm})$

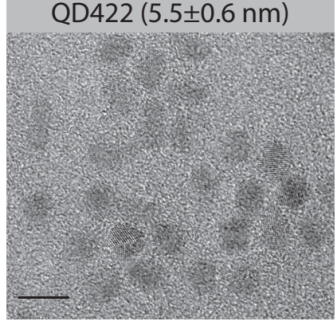

Figure S1. TEM images of the size series of ZnSe nanocrystals. Representative size-matched TEM images of $\mathrm{ZnSe}$ cores between 2.9 and $5.5 \mathrm{~nm}$ in diameter. The scale bar indicates $10 \mathrm{~nm}$ in all images. The average \pm standard deviation diameter of the nanoparticles is included in the inset text in the corresponding image. The size statistics are based on $n=126-236$ particles, depending on the sample. 

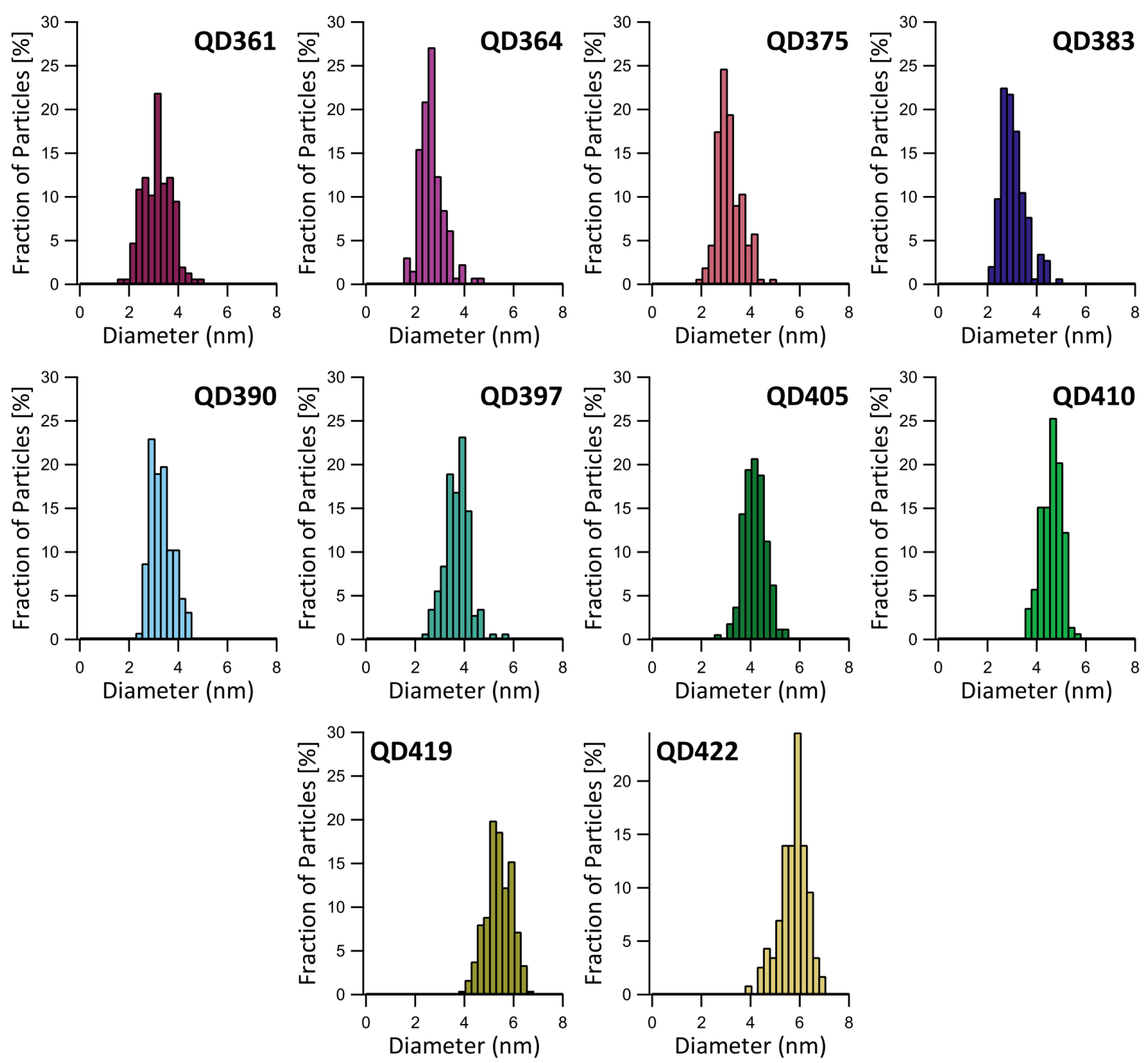

Figure S2. Histograms demonstrating size distribution of QD samples obtained through analysis of sizing based on TEM images. 


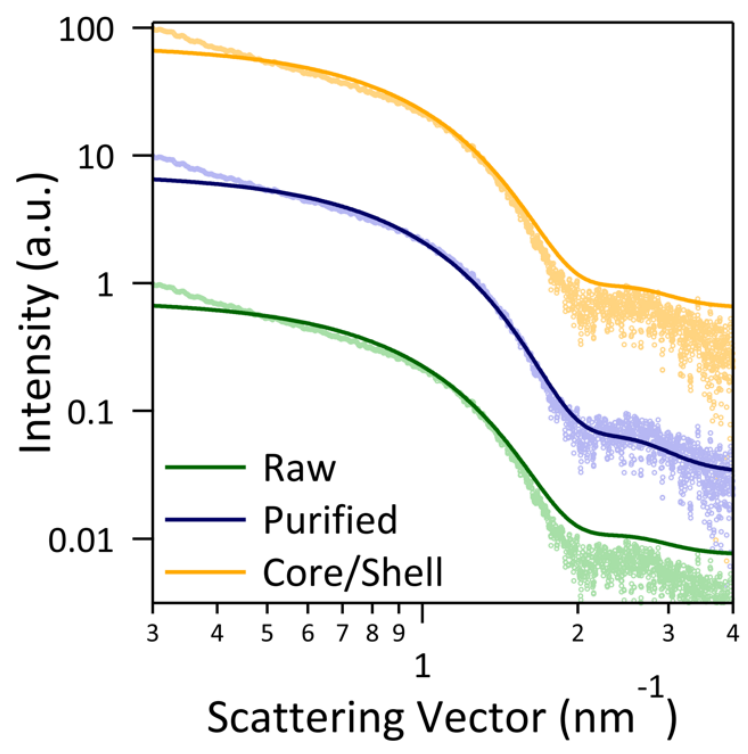

\begin{tabular}{|cc}
\hline Sample & Diameter (nm) \\
\hline Raw & $5.77 \pm 0.94$ \\
Purified & $5.70 \pm 0.94$ \\
Core/Shell & $5.74 \pm 0.98$ \\
\hline
\end{tabular}

Figure S3. Experimental SAXS patterns and average diameters obtained for QD422. SAXS measurements were performed on raw aliquots and purified solutions of QD422 prepared through precipitation and resuspension. The SAXS data was also modeled as a spherical core/shell heterostructure with all three patterns yielding comparable average diameters.

Table S1. Sample-specific data. Comparison of sizing analysis using small angle X-ray scattering (SAXS) and transmission electron microscopy (TEM). Absorption values used with MP-AES data from Table 1 for $\varepsilon$ calculations.

\begin{tabular}{|c|cc|ccc|c|c|}
\cline { 2 - 8 } \multicolumn{1}{c|}{} & \multicolumn{2}{c|}{ SAXS } & \multicolumn{3}{c|}{ TEM } & \multicolumn{2}{c|}{ Absorption } \\
\hline Sample & $\begin{array}{c}\text { Diameter } \\
(\mathbf{n m})\end{array}$ & $\begin{array}{c}\text { Std. Dev. } \\
(\mathbf{n m})\end{array}$ & $\begin{array}{c}\text { Diameter } \\
(\mathbf{n m})\end{array}$ & $\begin{array}{c}\text { Std. Dev. } \\
(\mathbf{n m})\end{array}$ & $\begin{array}{c}\text { No. QDs } \\
\text { Sized }\end{array}$ & $\mathbf{2 8 0} \mathbf{~ n m}$ & $\mathbf{1 S}$ Peak \\
\hline QD361 & 2.04 & 0.57 & 2.85 & 0.61 & 146 & 1.158 & 0.549 \\
QD364 & 2.21 & 0.58 & 2.41 & 0.38 & 134 & 1.217 & 0.577 \\
QD375 & 2.48 & 0.67 & 2.86 & 0.48 & 154 & 1.283 & 0.499 \\
QD383 & 2.86 & 0.67 & 2.77 & 0.62 & 142 & 1.302 & 0.499 \\
QD390 & 3.02 & 0.83 & 3.04 & 0.44 & 126 & 1.209 & 0.358 \\
QD397 & 3.46 & 0.48 & 3.42 & 0.51 & 142 & 1.245 & 0.402 \\
QD405 & 4.31 & 0.69 & 3.87 & 0.48 & 159 & 1.211 & 0.304 \\
QD410 & 4.42 & 0.70 & 4.30 & 0.38 & 138 & 1.184 & 0.291 \\
QD419 & 5.36 & 1.0 & 5.10 & 0.26 & 236 & 1.198 & 0.204 \\
QD422 & 5.75 & 0.87 & 5.46 & 0.58 & 114 & 1.206 & 0.197 \\
\hline
\end{tabular}



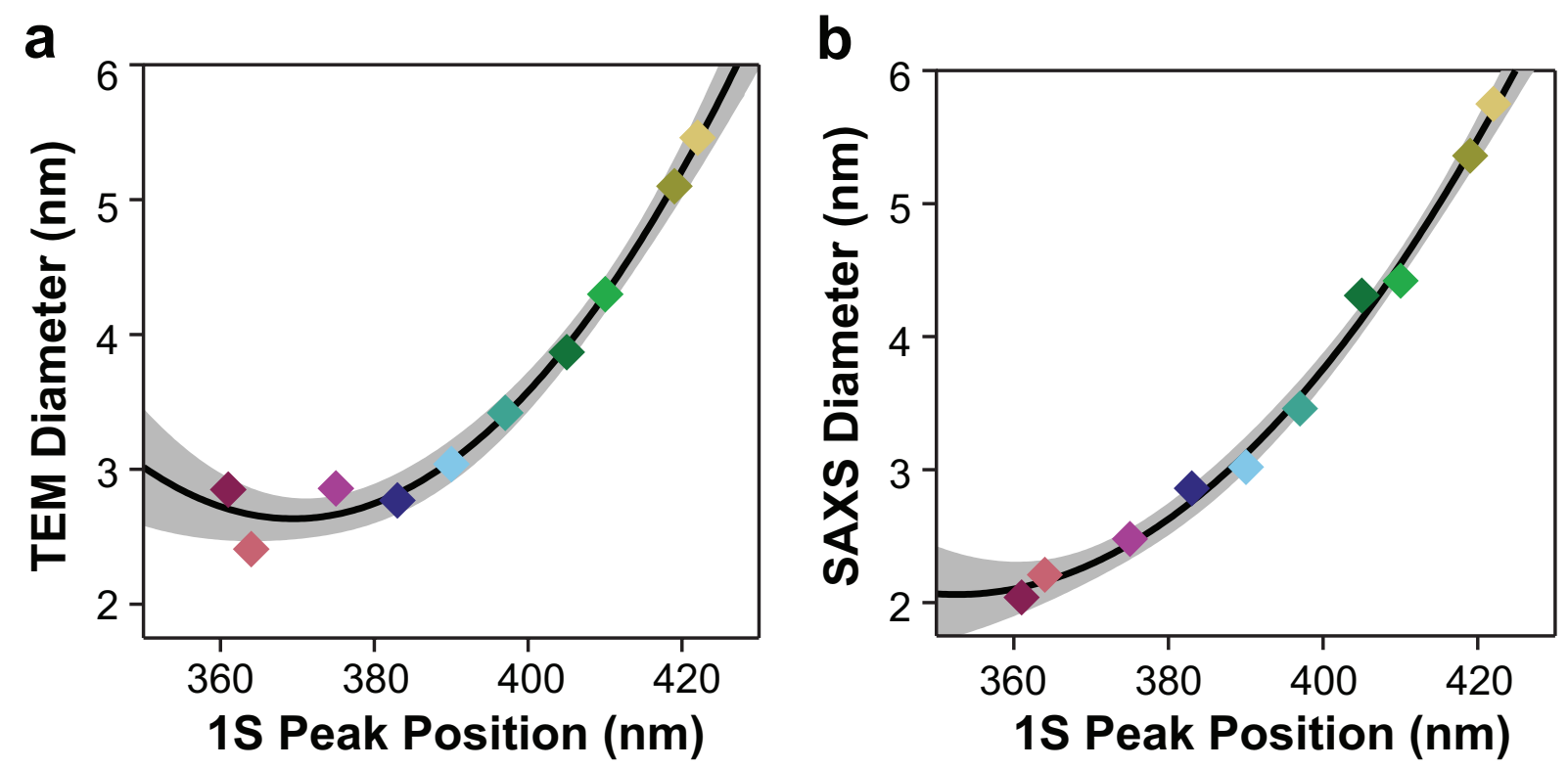

Figure S4. Mean diameter versus first excitonic absorption (1S) peak position. Comparing (a) mean TEM diameters to (b) mean SAXS diameters versus $1 \mathrm{~S}$ peak position indicates that the mean values increase monotonically for the SAXS diameters with respect to 1S peak position, whereas at low particle sizes, the TEM mean diameters are more variable, making the generation of effective (and physically realistic) fitting equations more challenging. Quadratic fits were used in both plots to guide the eye with the 95 percent confidence interval shaded in grey.
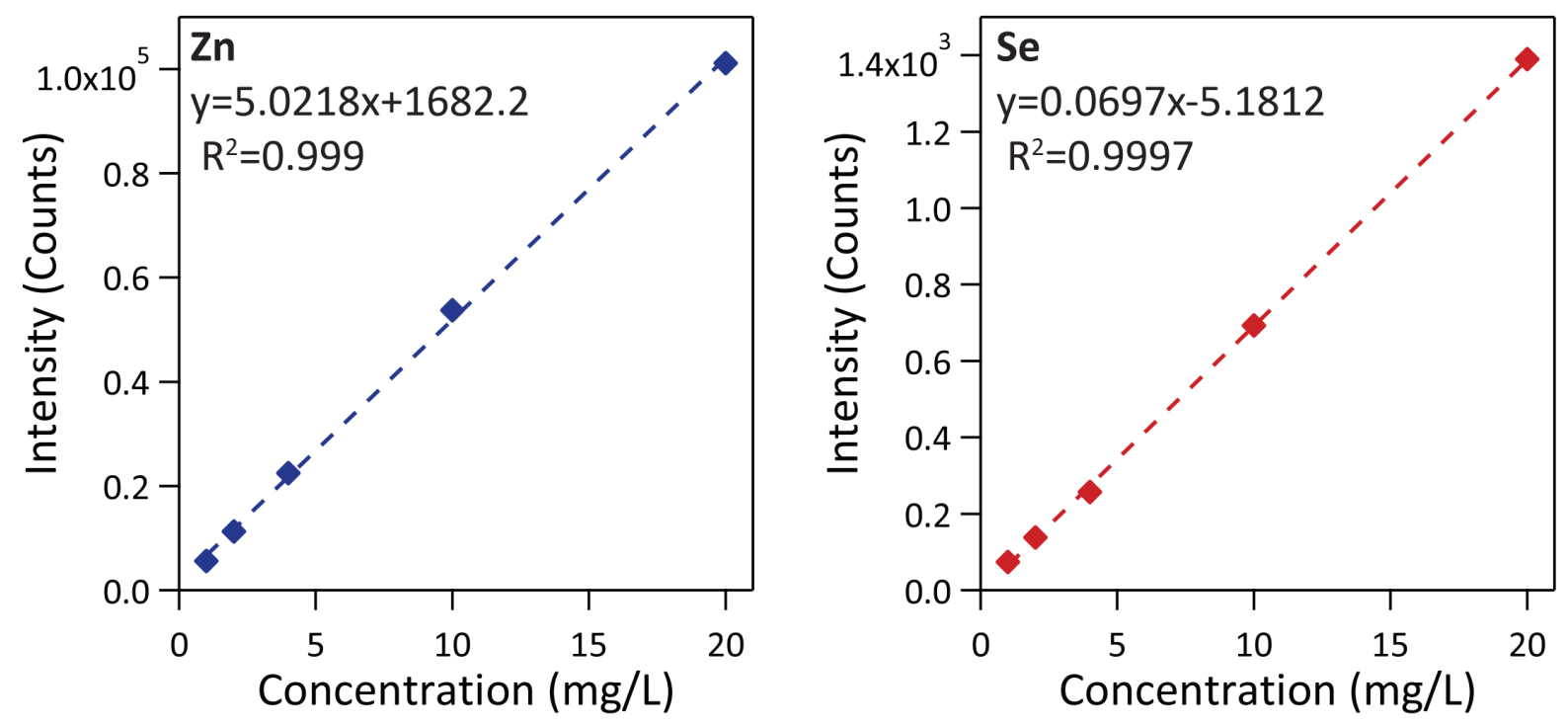

Figure S5. MP-AES calibration curves of $\mathrm{Zn}$ and Se ions. 
Table S2. Intrinsic absorption coefficient values.

\begin{tabular}{ccc}
\hline $\boldsymbol{\lambda}$ & $\bar{\mu}_{\boldsymbol{l}}\left(\mathbf{c m}^{\mathbf{- 1}}\right)$ & $\boldsymbol{\mu}_{\mathrm{i}, \mathbf{t h}} \mathbf{( \mathbf { c m } ^ { - 1 } )}$ \\
\hline $260 \mathrm{~nm}$ & 266,000 & 324,000 \\
$280 \mathrm{~nm}$ & 174,000 & 190,000 \\
$300 \mathrm{~nm}$ & 131,000 & 147,000 \\
\hline
\end{tabular}

Note S1. Using the intrinsic absorption coefficient to calculate zinc, selenium, and ZnSe unit concentration.

Combining Equations 9 and 10 produces an equation to use the high energy intrinsic absorption coefficients to calculate the concentration of zinc in the nanoparticles:

$$
C_{Z n}=\frac{A b s_{\lambda} \ln (10)}{\mu_{\mathrm{i}, \lambda} L} \frac{M_{Z n}}{M_{Z n S e}} \frac{2 \rho_{Z n S e}}{\left(1+\frac{1}{R_{Z n}}\right)}
$$

where $A b s_{\lambda}$ is the absorbance at a high energy wavelength $\lambda, \mu_{\mathrm{i}, \lambda}$ is the intrinsic absorption coefficient at $\lambda$ (Table S3), $L$ is the path length of light through the sample in $\mathrm{cm}$, and $R_{\frac{Z n}{S e}}$ is the elemental ratio of zinc to selenium in the particle. The equation simplifies by combining the constants for the mass of zinc $M_{Z n}=65.38 \mathrm{~g} / \mathrm{mol}$, the mass of zinc selenide $M_{Z n S e}=144.35$ $\mathrm{g} / \mathrm{mol}$, and the density of zinc selenide $\rho_{Z n S e}=5.27 \mathrm{~g} / \mathrm{cm}^{3}$ :

$$
\begin{aligned}
& \frac{\ln (10) M_{Z n} 2 \rho_{Z n S e}}{M_{Z n S e}}=11\left(\frac{\mathrm{g}}{\mathrm{mL}}\right) \\
& C_{Z n}\left(\frac{\mathrm{g}}{\mathrm{mL}}\right)=\frac{A b s_{\lambda}}{\mu_{\mathrm{i}, \lambda} L} \frac{11}{\left(1+\frac{1}{R_{\frac{Z n}{S e}}}\right)}
\end{aligned}
$$

The ratio of zinc to selenium $R_{\frac{z}{S e}}$ is a dependent on particle diameter $(D, \mathrm{~nm})$ and is described by Equation 4. Using this expression, the size-dependent concentration equation can be displayed to produce mass or molar concentrations, respectively:

$$
\begin{gathered}
C_{Z n}\left(\frac{\mathrm{g}}{\mathrm{mL}}\right)=\frac{A b s_{\lambda}}{\mu_{\mathrm{i}, \lambda} L} \frac{11}{\left(1+\frac{1}{\left(1+1.58 D^{-2.09}\right)}\right)} \\
C_{Z n}(\mathrm{M})=\frac{168 A b s_{\lambda}}{\mu_{\mathrm{i}, \lambda} L\left(1+\frac{1}{\left(1+1.58 D^{-2.09}\right)}\right)}
\end{gathered}
$$


If one assumes a $\mathrm{Zn}$ :Se molar ratio of 1.1 , which is the $R_{\frac{z n}{S e}}$ for a $3.5 \mathrm{~nm}$ diameter particle, the expression simplifies to require only the experimental inputs of absorbance at $\lambda$, the pathlength of light through the sample $(L, \mathrm{~cm})$, and the intrinsic absorption coefficient $\mu_{\mathrm{i}, \lambda}$ from Table S3:

$$
C_{Z n}\left(\frac{\mathrm{g}}{\mathrm{mL}}\right)=\frac{5.76 A b s_{\lambda}}{\mu_{\mathrm{i}, \lambda} L}
$$

Because these particles contain and excess of zinc, we assume that all Se is bound in $\mathrm{ZnSe}$ units, whereas some zinc is on the surface, extraneous to the $\mathrm{ZnSe}$ units. Applying the treatment above to the concentration of Se enables the determination of the selenium concentration from the high energy absorbance and, concomitantly, the determination of the ZnSe unit concentration:

$$
C_{S e}=\frac{A b s_{\lambda} \ln (10)}{\mu_{\mathrm{i}, \lambda} L} \frac{M_{S e}}{M_{Z n S e}} \frac{2 \rho_{Z n S e}}{\left(1+\frac{1}{R_{\frac{S e}{Z n}}}\right)}
$$

Aggregating the constants using $M_{S e}=78.96 \mathrm{~g} / \mathrm{mol}$ and noting that $\frac{1}{R_{\frac{S e}{Z n}}}=R_{\frac{Z n}{S e}}$

$$
\begin{gathered}
C_{S e}\left(\frac{\mathrm{g}}{\mathrm{mL}}\right)=\frac{13.28 A b s_{\lambda}}{\mu_{\mathrm{i}, \lambda} L\left(1+R_{\frac{Z n}{S e}}\right)} \\
C_{Z n S e}(\mathrm{M})=C_{S e}(\mathrm{M})=\frac{168 A b s_{\lambda}}{\mu_{\mathrm{i}, \lambda} L\left(1+R_{\frac{Z n}{S e}}\right)}
\end{gathered}
$$


Note S2. Confirmation of molar extinction coefficient self-consistency.

In order to confirm that the equations presented for the characterization of $\mathrm{ZnSe}$ nanoparticles presented here are self-consistent, the $\varepsilon_{1 \mathrm{~s}}$ values determined using either the band edge absorption or the high energy absorption equations were compared. The $1 \mathrm{~S}$ peak position $\left(\lambda_{1 \mathrm{~S}}\right)$ and absorbance intensity $\left(A b s_{1 S}\right)$ listed in Table $\mathbf{S 1}$ were used to calculate the molar extinction coefficient at the 1S peak $\left(\varepsilon_{1 S}\right)$ by first determining particle diameter using Equation 2:

and using that result in Equation 6:

$$
D(n m)=\frac{215.17}{\left(459-\lambda_{1 S}\right)}
$$

$$
\varepsilon_{1 S}=35,100 \cdot D^{1.71}
$$

The $\varepsilon_{1 S}$ can also be calculated from the $\varepsilon_{280}$ from Equation 12:

$$
\varepsilon_{280}=23,800 D^{3}
$$

using the $\left(A b_{280}\right)$, which is also listed in Table $\mathbf{S 1}$ :

$$
\varepsilon_{1 S}=\frac{\varepsilon_{280} A b s_{1 S}}{A b s_{280}}
$$

When these two approaches to calculating particle molar extinction coefficient are plotted against each other, their congruence can be easily visualized (Figure S6).

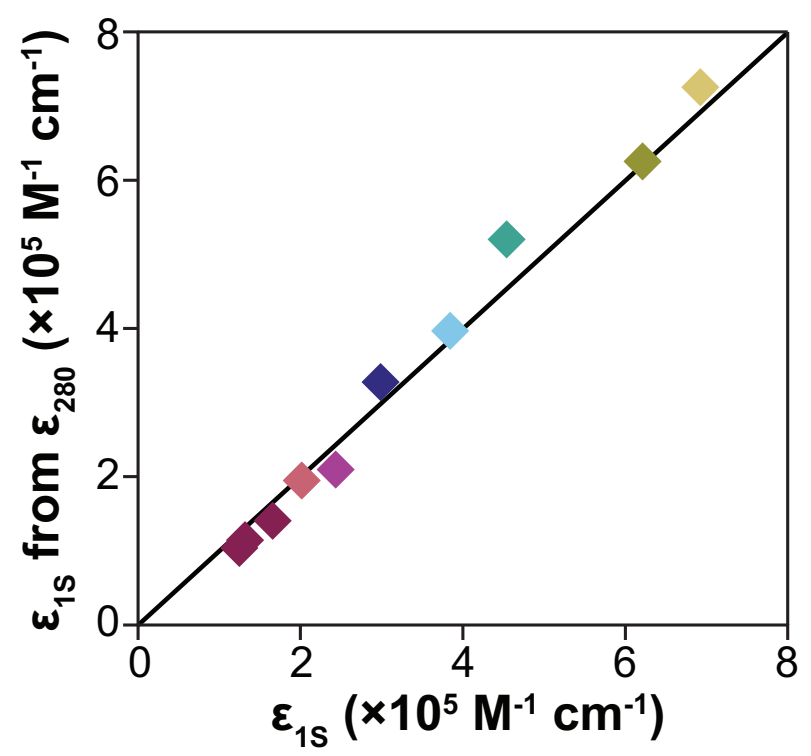

Figure S6. Confirmation of calculation consistency. 

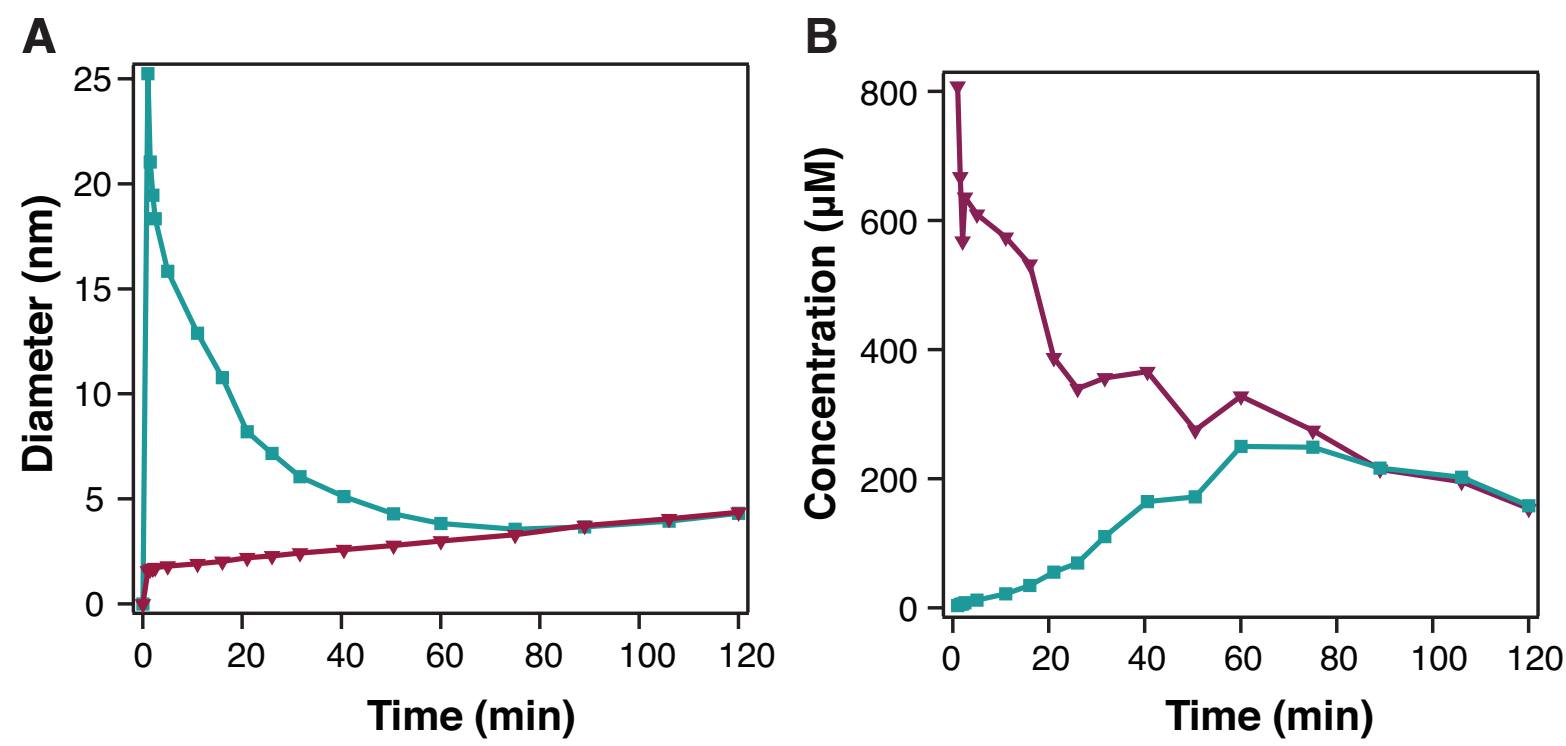

Figure S7. Reaction tracking comparing previously published and current equations. (A) ZnSe particle diameters calculated using Equation 2 (mauve triangles) and the previously published equation (teal squares). ${ }^{2}$ (B) Integrated molar extinction coefficient-based calculation of concentrations calculated using Equation 8 (mauve triangles) and the previously published equation (teal squares). ${ }^{2}$

\section{References:}

1. Li, J. \& Wang, L. W. Band-structure-corrected local density approximation study of semiconductor quantum dots and wires. Phys. Rev. B - Condens. Matter Mater. Phys. 72 , (2005).

2. Lin, S., Li, J., Pu, C., Lei, H., Zhu, M., Qin, H., and Peng, X. Surface and intrinsic contributions to extinction properties of ZnSe quantum dots. Nano Res. 13, 824-831 (2020).

3. Dorfs, D., Salant, A., Popov, I. \& Banin, U. ZnSe quantum dots within CdS nanorods: A seeded-growth type-II system. Small 4, 1319-1323 (2008). 